Research Article

\title{
A Novel Method for Integration of Online Educational Resources via Teaching Information Remote Scheduling
}

\author{
Kang Zhao \\ College of Literature and Media, Xi'an Fanyi University, Xi'an 710105, China \\ Correspondence should be addressed to Kang Zhao; zhaokang@xafy.edu.cn
}

Received 11 June 2021; Revised 21 July 2021; Accepted 25 July 2021; Published 4 October 2021

Academic Editor: Hussein Abulkasim

Copyright $\odot 2021$ Kang Zhao. This is an open access article distributed under the Creative Commons Attribution License, which permits unrestricted use, distribution, and reproduction in any medium, provided the original work is properly cited.

\begin{abstract}
The traditional online education resource integration technology ignores the calculation of the characteristic variance contribution rate of online educational resources, and the evaluation of educational resources is biased, which leads to the low ability of resource integration. Therefore, the online education resource integration technology based on remote scheduling of teaching information is proposed. Based on the theory of remote scheduling of teaching information, the quantitative value of online educational resources integration is obtained. By calculating the integration probability of online educational resources, the influencing factors of online educational resources in the process of integration are determined. The principle of teaching information remote scheduling is integrated into the feature vector extraction of online educational resources, and the contribution rate of feature variance of online educational resources is calculated. By extracting the number of main factors of the online educational resources feature vector, the feature vector of online educational resources is decomposed. Taking the wavelet entropy of online educational resources as the fusion weight of online educational resources, the integration result of online educational resources is obtained through wavelet transform. The experimental results show that the online education resource integration technology based on remote scheduling of teaching information has ideal application performance in integration probability, the goodness of fit, and robustness.
\end{abstract}

\section{Introduction}

The development and progress of education informatization have contributed to the accumulation of more and more information resources in the education information system $[1,2]$. However, due to factors such as the level of technology and management system, islands of information exist in online education information resources. To solve this problem, online educational resources need to be developed and integrated in a rational way, so that these resources serve for online education [3].

In order to improve the capability in retrieving educational resources, the literature [4] analyzed the core structure model of multimedia resource distribution in embedded distance education networks, sorted the nonlinear time of educational resource information flow, divided the time window of multimedia resources into a set of time slots uniformly distributed using the smoothing filter method, and then explored the accessibility of multimedia resources in distance education, through which the balance between resource allocation and resource utilization were effectively improved. The literature [5] points out that, in the traditional method, there was no statistics on the number of specific departments in computing resource integration, which led to the low accuracy of integration; it proposes the method of integrating educational resources for college students based on the factor analysis theory, so as to obtain many empirical values, quantify these values, and get the main influencing elements of resource integration, while based on the factor analysis theory and the influence on the integration of educational resources of college students, the coupling matrix is obtained for the integration of the main educational resources of college students, and the number of basic departments is calculated through the integration of these resources, so as to analyze and study the current situation of the integration of educational resources of 
college students, and it has been proved by experiment that this method has good robustness and can better integrate the educational resources for students.

Based on the above research background, this paper applies the remote scheduling of teaching information to the integration of online educational resources, which improves the ability of online educational resources integration.

\section{Online Education Resource Integration Technology}

2.1. Influencing Factors of Online Educational Resources in the Process of Integration. In the process of integration of online educational resources, based on the theory of remote scheduling of teaching information [6], different quantitative values existed in the integration of online educational resources were obtained to extract the factors influencing the integration of online educational resources as follows.

Suppose that $s$ denotes the factor of multifaceted conflicts that influence the integration of online educational resources, $x$ denotes the abnormal measurement value in the process of integration of online educational resources, $n$ denotes the time frame in the process of integration of online educational resources, and $j$ denotes the quantitative value of the integration performance of online educational resources. Combined with the theory of remote scheduling of teaching information [7], the quantitative value of the integration of online educational resources generated in the process of integration of online educational resources due to the expectation discrepancy that educational resources have is obtained and calculated as follows:

$$
O_{h}=\sum_{j=1}^{n} \frac{s}{x_{j}}+\sum_{j=1}^{n} \frac{c_{k h} \times v_{s}}{x_{j}},
$$

where $c_{k h}$ denotes the regular performance of online educational resources in the integration process and $v_{s}$ denotes the adaptive capacity shown by online educational resources in the integration process. Then the integration probability of online educational resources can be calculated using the following equation:

$$
\vartheta\left(V_{y}\right)=\frac{\mu_{b} \times \kappa_{r}}{x_{j}} c_{k h} O_{h}
$$

where $\kappa_{r}$ denotes the current status of integration of online educational resources and $\mu_{b}$ denotes the characteristics of changes in the process of integration of online educational resources.

Suppose that $h_{r}$ denotes the characteristics of online educational resources to determine the influencing factors of online educational resources in the process of integration as follows:

$$
K_{v}=\frac{h_{r} \times z_{o}}{\tau_{m}} \times \frac{F^{D}}{\iota(i) \cdot a_{d}},
$$

where $\iota(i)$ denotes the attributes of online educational resources, $i$ denotes the attribute vector of factors influencing the integration of online educational resources, $F^{D}$ denotes the features selected, $z_{o}$ denotes the time required in the process of integration of online educational resources, $\tau_{m}$ denotes the probability of failure in the integration of online educational resources, and $a_{d}$ denotes the integration cycle of online educational resources.

Based on the theory of remote scheduling of teaching information, the quantitative value of online educational resources integration is obtained. By calculating the integration probability of online educational resources, the influencing factors of online educational resources in the process of integration are determined.

\subsection{Extraction of Online Educational Resources Feature} Vector. In the process of integration of online educational resources, the principle of teaching information remote scheduling is integrated into the feature vector extraction of online educational resources [8], and the contribution rate of feature variance of online educational resources is calculated. The observable random vector of online educational resource features is given. By extracting the number of main factors of online educational resources feature vector as follows, the feature vector of online educational resources is decomposed [9].

Suppose that $n$ denotes the number of feature variables of the original online educational resources, $X$ denotes the condition of $X=x_{1}, x_{2}, \ldots, x_{n}$ that the $n$ feature variables in the original online educational resources samples need to satisfy to carry out orthogonal transformation of online educational resources [10] and to integrate $u^{\prime}$ online educational resources feature variables $\left(y_{1}, y_{2}, y_{3}\right)$, and $R$ denotes the correlation coefficient matrix of online educational resources samples. The theory of remote scheduling of teaching information [11] is used to establish the feature equation of online educational resources as follows:

$$
\lambda^{n}(i)=\frac{\{R \otimes X\}^{n}}{\left\{\left(y_{1}, y_{2}, y_{3}\right)\right\}} \otimes \frac{\left(x_{1}, x_{2}, \ldots, x_{n}\right)}{u^{\prime}} \text {. }
$$

Suppose that $\lambda_{i}$ denotes the number of nonnegative feature values of the correlation coefficient matrix of online educational resources samples. Under the condition that satisfies $\lambda_{1} \geq \lambda_{2} \geq \lambda_{n} \geq 0, \lambda_{i}$ sorting is conducted, and then the first $m$ online educational resource features can be extracted as follows:

$$
\Phi(p)=\frac{m \otimes\left(\lambda_{i}\right)}{\xi(e)} \otimes \eta(r),
$$

where $\xi(e)$ denotes the noise interference of online educational resources feature and $\eta(r)$ denotes the uncertainty of online educational resources feature vector.

Suppose that $\alpha$ denotes the variance of the first $m$ online educational resources features. The calculating formula is as follows:

$$
\beta(p)=\frac{m \otimes \alpha}{\mu(R)} \times \nu(e)\left(\sigma^{*} \otimes \kappa\right)
$$

where $\mu(R)$ denotes the weights of online educational resources feature samples, $\nu(e)$ denotes the information 
entropy of different online educational resource features, $\sigma^{*}$ denotes the optimal threshold value of online educational resource feature variables, and $\kappa$ denotes the observed variables of student features.

$X=x_{1}, x_{2}, \ldots, x_{n}$ is defined as the random vector of online educational resource features, $a_{i j}$ denotes the factor loading of online educational resources feature vector, and the observable random vector of online educational resource features is calculated as follows:

$$
\partial(X)=\frac{X \otimes F}{\left(a_{i j}\right)_{n \mp m}} \otimes c_{i} \otimes \varepsilon_{i} \times X_{i},
$$

where $F$ denotes the unobservable vector of online educational resources, $c_{i}$ denotes the factor loadings of particular online educational resources, and $\varepsilon_{i}$ denotes the unique factors affecting factor loadings $c_{i}$.

The degree of uncertainty of the online educational resources information source $X^{*}$ is measured by the equation as follows:

$$
H\left(X^{*}\right)=\frac{p_{i} \log p_{i}}{H\left(p_{1}, p_{2}, \ldots, p_{n}\right)} I\left(a_{i}\right),
$$

where $I\left(a_{i}\right)$ denotes the space of online educational resources information source $X^{*}, p_{i}$ denotes the probability of occurrence of online educational resources integration strategy, and $H\left(p_{1}, p_{2}, \ldots, p_{n}\right)$ denotes the probability of occurrence of discrete random variables.

Suppose that $I\left(\lambda_{i}\right)$ denotes the information function of online educational resources integration. The probability of online educational resources integration behavior can be defined as

$$
\xi(w)=\frac{I\left(\lambda_{i}\right) \otimes H\left(X^{*}\right)}{\zeta(k)} \otimes \vartheta(R),
$$

where $\zeta(k)$ denotes the information volume of online educational resources and $\vartheta(R)$ denotes the cumulative information contribution rate of online educational resources integration.

According to the abovementioned, the principle of remote scheduling of teaching information is integrated into the feature vector extraction of online educational resources, the contribution rate of feature variance of online educational resources is calculated, and by extracting the number of main factors of the feature vector of online educational resources, the feature vector of online educational resources is decomposed, which lays the foundation for the integration of online educational resources.

\subsection{Integration of Online Educational Resources.} According to the feature vector extraction results of online educational resources, a complete online educational resources dataset is established. Through data fusion algorithm [12], all the features of online educational resources in the online educational resources dataset are reconstructed [13], and then, the reconstructed online educational resources dataset is decomposed. The wavelet variance of online educational resources is calculated according to the decomposition result [14], which is used as the weight fusion wavelet coefficient to obtain the integration result of online educational resources.

Suppose that $q$ explorers manage multiple online educational resources $X$ simultaneously. The value of the online educational resources reserve in each explorer can be expressed as

$$
Z_{i}=X+V_{i}, \quad i=1,2, \ldots, m,
$$

where $V_{i}$ denotes the parameters of online educational resources in the explorers.

Suppose that $\delta_{i}$ denotes the weight in each explorer, $\sigma_{i}$ denotes the variance of parameters of online educational resources, the integration value of online educational resources finally obtained is $Z=\sum_{i=1}^{q} \delta_{i} Z_{i}$, and $\sum_{i=1}^{q} \delta_{i}=1$, $0<\delta_{i}<1$. The total mean square error of online educational resources integration is as follows:

$$
\sigma^{2}=E\left[(X-Z)^{2}\right]=\sum_{i=1}^{q} \delta_{i}^{2} \sigma_{i}^{2}
$$

According to the theory for the extremum of multivariable functions [15], the minimum value of equation (11) can be calculated as $1 / \sum_{i=1}^{q} 1 / \sigma_{i}^{2}$, and the weighting factor corresponding to the minimum value is

$$
\delta_{i}=\frac{1 / \sigma_{i}^{2}}{\sum_{i=1}^{q} 1 / \sigma_{i}^{2}} .
$$

The weighting factor obtained from equation (12) is used to reconstruct the features of online educational resources [16], and the equation is

$$
Z_{J}=\sum_{k} h_{n}(J, k) \varphi_{J, k}+\sum_{J} \sum_{k} g_{n}(J, k) \psi_{J, k},
$$

where $Z_{J}$ denotes the reconstructed online educational resources, $\varphi_{J, k}$ denotes the scaling function of online educational resources at layer $J, h_{n}(J, k)$ denotes the $k^{\text {th }}$ approximate coefficient of online educational resources, $\psi_{J, k}$ denotes the wavelet resource function of online educational resources at layer $J$, and $g_{n}(J, k)$ denotes the $k^{\text {th }}$ detail resource function of online educational resources.

Suppose that $W_{h, i}$ denotes the weighting factors of the approximate parameters of online educational resources and $W_{g, i}$ denotes the weighting factors of the detail parameters of online educational resources. Then it can be obtained as follows:

$$
\sigma^{2}=\sigma_{h}^{2}+\sigma_{g}^{2}=E\left[Z_{V}^{2}-\sum_{i=1}^{q} W_{h, i}^{2} \frac{1}{2} \eta_{i} \varphi_{J, k}\right] .
$$

According to equation (14), it can be known that the mean variance of the weighted results of online educational resources integration is less than or equal to that of the optimal weighting of online educational resources in the time domain, and the remote scheduling of online teaching resources through teaching information [17] leads to an increasingly smaller mean variance of online educational resources with the decomposition of the scale, and the final 
conclusion provides a theoretical basis for the integration of online educational resources [18].

Suppose that $X$ denotes the parameter sequence of online educational resources managed. Based on the teaching information remote scheduling theory, it can be known that wavelet transform has the property of energy conservation under orthogonal wavelet basis, and based on the time sequence [19], the energy can be divided on the scale domain, and using the wavelet basis function, the energy on the explorers of online educational resources can be decomposed as

$$
\|X\|^{2}=\sum_{i=1}^{J}\left\|W_{J}\right\|^{2}+\left\|V_{J}\right\|^{2}
$$

The measured variance of online educational resources is given by

$$
\sigma_{X}^{2}=\frac{1}{N}\left\|\sum_{N=1}^{N}(X-\bar{X})^{2}\right\|=\left(\left\|W_{j, n}\right\|^{2}+\left\|V_{j, n}\right\|^{2}\right) .
$$

As $V_{j}$ approximates $\bar{X}$, the wavelet variance and the average wavelet energy on scale $j$ in equation (16) are normalized as follows:

$$
p_{j}(E)=\frac{E_{j}}{E}=\frac{1 / N\left\|W_{j}\right\|^{2}}{E},
$$

where $E$ denotes the total energy of online educational resources, $E=\sum_{j=1}^{J} E_{j}$, and the energy sequence $p_{j}(E)$ of online educational resources normalized denotes the ratio of the energy of online educational resources to the total energy. The wavelet entropy of online educational resources is obtained based on the distribution of the energy sequence of online educational resources [20-22]. The specific procedure is as follows:

$$
H_{w e}=H(P)=H\left(p_{1}(E), p_{2}(E), \ldots, p_{J}(E)\right) .
$$

To sum up, taking the wavelet entropy of online educational resources as the fusion weight of online educational resources, the integration result of online educational resources is obtained through wavelet transform, realizing the integration of online educational resources.

\section{Experimental Comparative Analysis}

An experiment was conducted to verify the effectiveness of online educational resources integration technology based on remote scheduling of teaching information. Six thousand three hundred and twenty-five types of online educational resources in varied majors of a university were selected as objects of the experiment, from which 700 types were chosen for integration. The experiment results were subject to check analysis.

Tests were conducted with the online educational resources integration technology based on teaching information remote scheduling, and the integration technologies of online educational resources in the literature $[4,5]$. The results obtained from equation (2) are used to compare the

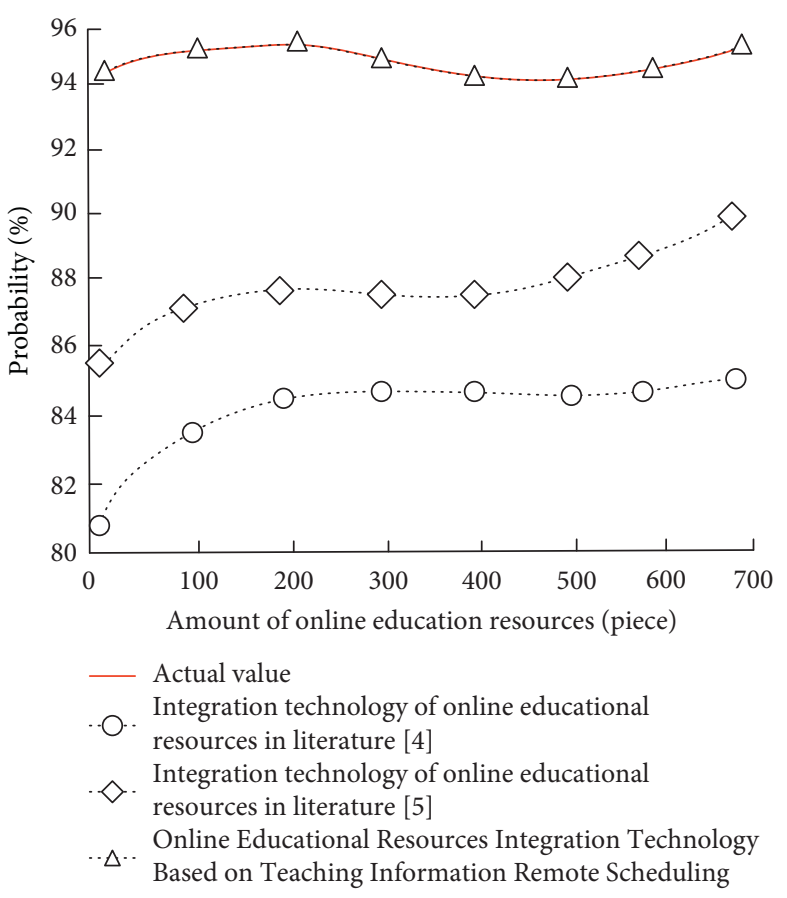

FIGURE 1: Comparison results of online educational resources integration probability.

integration probability and the actual value of the three integration technologies, which are shown in Figure 1.

It can be seen from the results in Figure 1 that the probability of having influencing factors on online educational resources integration technology based on teaching information remote scheduling coincides with the actual value. The reason is that the technology allows beforehand calculation of the probability of factors having influence on online educational resources integration to extract features of online educational resources, based on which the online educational resources integration probability matches the actual value.

To further prove the rationality of online educational resources integration technology based on teaching information remote scheduling, the goodness of fit of the technology is verified through relevant checking residuals by using the following equation:

$$
R^{\text {new }}=1-\frac{Q}{2 \sum \widehat{y}_{2}} \times 100 \%,
$$

where new denotes the coefficient of determination, $\hat{y}_{2}$ denotes the fitting degree, and $Q$ denotes the residual sum of squares.

The test results of goodness of fit by online educational resources integration analysis based on three different technologies are shown in Figure 2.

It is shown in Figure 2 that the online educational resources integration technology based on teaching information remote scheduling is superior to the other two technologies in terms of goodness of fit because the quantitative value of online education resources integration features is obtained based on the technology, presenting the 


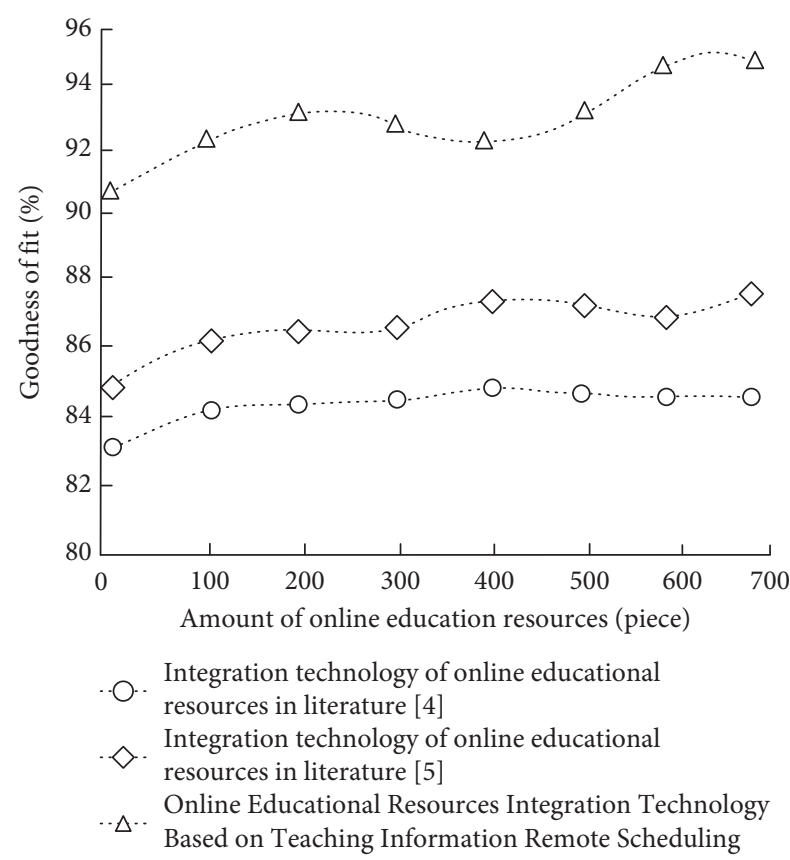

Figure 2: Test results of goodness of fit by online educational resources integration analysis.



FIGURE 3: Robustness of online educational resources integration.

correlation between influencing factors on online educational resources integration and online educational resources integration. Thus, the technology shows a better goodness of fit.

Based on the results above, the robustness of the three online educational resources integration technologies was tested, and the results are as shown in Figure 3.

It can be seen from Figure 3 that the online educational resources integration technology based on teaching information remote scheduling shows a better robustness than the other two technologies. The reason is that this technology analyzes the relation between the influencing factors on online educational resources and online educational resources, determining the integration degree of influencing factors on online educational resources. Thus, such technology has a better robustness.

\section{Conclusion}

A research on online educational resources integration technology based on teaching information remote scheduling is put forth in this paper. By determining influencing factors on online educational resources integration, the feature vector of online educational resources integration is extracted, and online educational resources are integrated pursuant to the online educational resources integration process. The results show that this technology can effectively improve the integration capability of online educational resources.

\section{Data Availability}

All the data can be requested from the corresponding author upon reasonable request.

\section{Conflicts of Interest}

The author declares no conflicts of interest.

\section{References}

[1] J. Wolfe and G. Hatsidimitris, "Physclips: multimedia, multilevel learning, and teaching resources," Journal of the Acoustical Society of America, vol. 132, no. 3, p. 2011, 2012.

[2] T. Okemakinde, S. O. Adedeji, and J. Ssempebwa, "Utilization of teaching resources and academic performance in technical colleges in Oyo state, Nigeria," Monthly Notices of the Royal Astronomical Society, vol. 380, no. 2, pp. 741-748, 2012.

[3] C. Klupiec, S. Pope, R. Taylor, D. Carroll, M. Ward, and P. Celi, "Development and evaluation of online video teaching resources to enhance student knowledge of livestock handling," Australian Veterinary Journal, vol. 92, no. 7, pp. 235-239, 2014.

[4] M. Nong, "Research on multimedia resource scheduling technology in distance education network," Modern Electronics Technique, vol. 40, no. 24, pp. 68-70, 2017.

[5] X.-Y. Qiao, "Optimization of college students' educational resources integration," Computer Simulation, vol. 34, no. 8, pp. 239-242, 2017.

[6] T. Wilkinson, "Primal pictures anatomy teaching resources: 3D anatomy software and 3D real-time viewer," Journal of Anatomy, vol. 220, no. 1, pp. 118-119, 2011.

[7] M. L. Aikens and E. L. Dolan, "Teaching quantitative biology: goals, assessments, and resources," Molecular Biology of the Cell, vol. 25, no. 22, pp. 3478-3481, 2014.

[8] S. B. Issenberg, S. Pringle, R. M. Harden, S. Khogali, and M. S. Gordon, "Adoption and integration of simulation-based learning technologies into the curriculum of a UK undergraduate education programme," Medical Education, vol. 37, no. s1, pp. 42-49, 2003. 
[9] C. C. Maponga and S. A. Feresu, "A community-based clinical pharmacy teaching, research, and service program in Zimbabwe," Pharmacotherapy: The Journal of Human Pharmacology and Drug Therapy, vol. 15, no. 5, pp. 614-620, 2012.

[10] I. A. Furtado, L. L. Neto, and A. J. Gonçalves-Ferreira, "Paths for anatomy education in the digital era: options of the institute of anatomy of the faculty of medicine of lisbon," Annals of Medicine, vol. 51, no. s1, p. 54, 2019.

[11] S. Pullen and K. Brinkert, "SolEn for a sustainable future: developing and teaching a multidisciplinary course on solar energy to further sustainable education in chemistry," Journal of Chemical Education, vol. 91, no. 10, pp. 1569-1573, 2014.

[12] M. Gupta and P. Lata, "Development of one-dimensional KLM model based on ABCD matrices," Diagnostic Ultrasound Imaging: Inside Out, vol. 8, no. 13, pp. 787-790, 2014.

[13] M. Stead, R. Stradling, M. Macneil et al., "Bridging the gap between evidence and practice: a multi-perspective examination of real-world drug education," Drugs: Education, Prevention \& Policy, vol. 17, no. 1, pp. 1-20, 2010.

[14] R. S. Wigton, "Use of linear models to analyze physicians' decisions," Medical Decision Making, vol. 8, no. 4, pp. 241-252, 1988.

[15] A/RES, "Towards full integration of persons with disabilities into society: a continuing world programme of action: resolution/adopted by the general assembly," Anaesthesia, vol. 65 , no. 8 , pp. 867-871, 2012.

[16] M. A. M. Arthur, R. Battat, and T. F. Brewer, "Teaching the basics: core competencies in global health," Infectious Disease Clinics of North America, vol. 25, no. 2, pp. 347-358, 2011.

[17] T. S. Klitzner, L. A. Rabbitt, and R.-K. R. Chang, "Benefits of care coordination for children with complex disease: a pilot medical home project in a resident teaching clinic," The Journal of Pediatrics, vol. 156, no. 6, pp. 1006-1010, 2010.

[18] S. Alsaggaf, S. S. Ali, N. N. Ayuob, B. S. Eldeek, and A. Elhaggagy, "A model of horizontal and vertical integration of teaching on the cadaveric heart," Annals of Anatomy-Anatomischer Anzeiger, vol. 192, no. 6, pp. 373-377, 2010.

[19] M. I. Kleib, "Integration of health informatics in baccalaureate nursing education: effectiveness of face-to-face vs. online teaching methods," Mathematische Zeitschrift, vol. 263, no. 4, pp. 1279-1296, 2012.

[20] I. L. Johnson, F. E. Scott, N. P. Byrne, K. A. MacRury, and J. Rosenfield, "Integration of community health teaching in the undergraduate medicine curriculum at the university of toronto," American Journal of Preventive Medicine, vol. 41, no. 4-supp-S3, pp. S176-S180, 2011.

[21] S. Mlambo, P. Rambe, and L. Schlebusch, "Effects of Gauteng province's educators' ICT self-efficacy on their pedagogical use of ICTS in classrooms," Heliyon, vol. 6, no. 4, Article ID e03730, 2020.

[22] A. Pakdaman-Savoji, J. C. Nesbit, and N. Gajdamaschko, "The conceptualisation of cognitive tools in learning and technology: a review," Australasian Journal of Educational Technology, vol. 35, no. 2, pp. 1-24, 2019. 